\title{
Transverse colon adenocarcinoma with direct cutaneous extension: complex multidisciplinary surgical management
}

https://doi.org/10.1515/jom-2022-0015

Received January 16, 2022; accepted February 2, 2022; published online February 23, 2022

A 66-year-old cachectic female presented to the emergency department in August 2021 with dizziness. Physical examination revealed a 3-4 cm infraumbilical ulcerative mass, present for approximately 1 month. CT scan showed an $8 \mathrm{~cm}$ lower abdominal mass contiguous with the bladder dome, involving the mid-transverse colon and several segments of small bowel without evidence of metastatic disease (Figure 1).

Percutaneous biopsy revealed poorly-differentiated adenocarcinoma, initially thought to be an urachal primary. The patient progressively developed anemia, bacteremia, and feculent discharge from her abdominal lesion (Figure 2). Following a multidisciplinary discussion, she was determined not to be a candidate for neoadjuvant chemotherapy. The decision was made to proceed with open surgical resection after her acute hematologic and infectious issues were addressed. She underwent an en bloc resection of the anterior abdominal wall, urachus, bladder dome, right hemicolectomy, with an ileocolic anastomosis and proximal loop diverting ileostomy. She required a complex abdominal wall closure with component separation, biologic mesh with vacuum-assist device and delayed skin grafting. Final pathology revealed locally advanced pT4bNO

*Corresponding author: Zachary A. Snow, DO, Main Line Health Urology Residency, 130 S. Bryn Mawr Avenue, 2nd Floor - A Wing, Bryn Mawr, PA, 19010, USA, E-mail: snowz@mlhs.org

Barak N. Marshall, OMS III, Philadelphia College of Osteopathic Medicine, Philadelphia, PA, USA

Paulette Dreher, DO, Main Line Health Urology Residency, Bryn Mawr, PA, USA

Samir N. Parikh, MD, Main Line Health Department of Colorectal Surgery, Media, PA, USA

Adam Walchak, MD, Main Line Health Department of Plastic and Reconstructive Surgery, Media, PA, USA

David B. Cahn, DO, Main Line Health Department of Urology, Media, PA, USA transverse colonic adenocarcinoma with direct extension into the bladder and small intestine. She is planning to undergo FOLFOX adjuvant chemotherapy.

The incidence of transverse colon cancer is less common than cancers of other segments of large bowel, comprising only $10 \%$ [1]. Additionally, locally advanced colon cancer with direct cutaneous extension is exceedingly rare, with most of the literature arising from isolated case reports [2-4]. Given the lack of randomized clinical trials available, challenging oncologic presentations should require multidisciplinary management to guide individualized care.

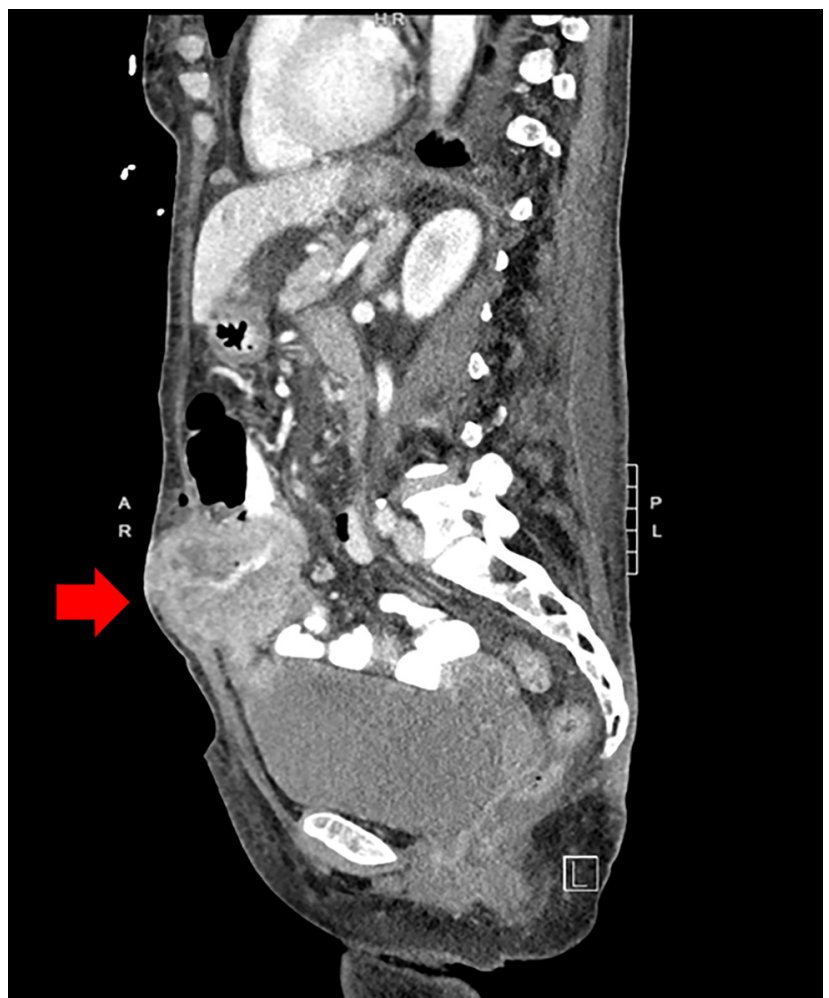

Figure 1: CT of the abdomen and pelvis with IV and oral contrast; sagittal view. Red arrow: $8 \mathrm{~cm}$ abdominal mass involving the bladder, transverse colon, and small bowel. 


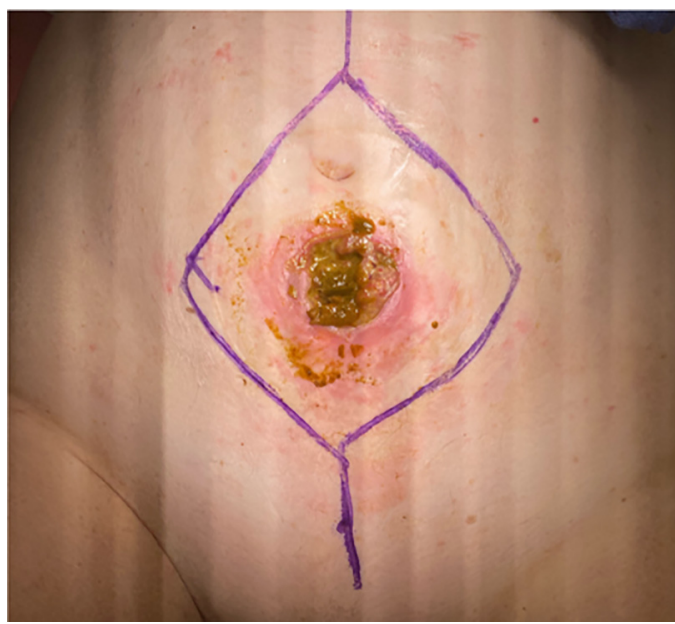

Figure 2: Ulcerating infraumbilical mass with feculent drainage (the outline represents palpable margins).

Research funding: None reported.

Author contributions: All authors provided substantial contributions to conception and design, acquisition of data, or analysis and interpretation of data; all authors drafted the article or revised it critically for important intellectual content; all authors gave final approval of the version of the article to be published; and all authors agree to be accountable for all aspects of the work in ensuring that questions related to the accuracy or integrity of any part of the work are appropriately investigated and resolved.

Competing interests: None reported.

\section{References}

1. Roy MK, Pipara A, Kumar A. Surgical management of adenocarcinoma of the transverse colon: what should be the extent of resection? Ann Gastroenterol Surg 2020;5: 24-31.

2. Tenreiro N, Ferreira C, Silva S, Marques R, Ribeiro A, Sousa PJ, et al. Locally advanced colon cancer with cutaneous invasion: case report. BMC Res Notes 2017;10:113.

3. Achanta CR, Dahiya D, Garg M, Rana SS, Behera A. Education and Imaging. Gastrointestinal: colon cancer with cutaneous infiltration. J Gastroenterol Hepatol 2013;28:762.

4. Freeman HP, Oluwole SF, Ganepola GA. Unusual presentations of carcinoma of the right colon. Cancer 1979;44:1533-7. 